\title{
КАЧЕСТВЕННОЕ ИССЛЕДОВАНИЕ МОДЕЛЬНОГО УРАВНЕНИЯ ЛЬЕНАРА ДЛЯ ОПИСАНИЯ АПЕРИОДИЧЕСКОГО ПРОФИЛЯ УЕДИНЕННОЙ ВОЛНЫ
}

\author{
(Представил Н. Алумяэ)
}

1. В настоящее время много внимания уделяется исследованию волновых процессов в активной среде $\left[{ }^{1,2}\right]$. Традиционный подход основывается на применении математических моделей параболического типа, которые могут быть описаны, например, системой $\left[{ }^{3}\right]$

$$
\frac{\partial E_{i}}{\partial t}=G_{i}(\vec{E})+D_{i} \nabla^{2} E_{i}, \quad i=1,2, \ldots, n
$$

Здесь $\vec{E}=\left(E_{i}\right)$ - переменные, определяющие состояние системы, $G_{i}-$ нелинейные функции, $D_{i}-$ коэффициенты диффузии. Существует много задач, приводимых к системе (1.1) - задача распространения нервного импульса $\left[{ }^{1,3-6}\right]$ или возбуждения в сердечной мышце $\left[{ }^{3}\right]$, реакция Белоусова-Жаботинского $\left[{ }^{2,3}\right]$, задача распространения генов в популяции $\left[{ }^{7}\right]$ и др. Исследование поведения системы (1.1) в общем случае сводится к изучению соответствующих уравнений стационарных волн, т. е. к изучению свойств обыкновенных нелинейных дифференциальных уравнений $\left[{ }^{1,6}\right]$.

Существует, однако, и другой класс задач, в котором волновой и диффузионный характеры процесса имеют одинаковое значение. В этом случае взамен системы (1.1) имеем

$$
\tau_{i}\left(\partial^{2} E_{i} / \partial t^{2}\right)+\partial E_{i} / \partial t=G_{i}(\vec{E})+D_{i} \nabla^{2} E_{i}, \quad i=1,2, \ldots, n,
$$

где $\tau_{i}-$ время релаксации. Нас интересует случай, когда только одно переменное $E_{j}$ имеет волновой характер, т. е. $\tau_{j} \neq 0, \tau_{i}=0, i \neq j$.

Математическая модель распространения нервного импульса берет свое начало из телеграфных уравнений. Как правило, при этом пренебрегается индуктивностью и в результате процесс описывается системой типа (1.1) $\left[{ }^{1,3-6}\right]$. K этой системе принадлежат известные модели Ходжкина-Хаксли $\left[{ }^{4,5}\right]$ и ФитцХью-Нагумо $\left[{ }^{5,6}\right]$. Х. М. Либерштейн, однако, предлагал сохранить индуктивность в телеграфных уравнениях и решил систему типа (1.2) численно $\left[{ }^{8}\right]$. Ю. Энгельбрехтом было предложено на основе полной (гиперболической) системы телеграфных уравнений эволюционное уравнение, описывающее распространение импульса в нервном волокне $\left[{ }^{9,10}\right]$. В удобно выбранных переменных это эволюционное уравнение имеет вид

$$
\left(\partial^{2} z / \partial \xi \partial x\right)+f(z)(\partial z / \partial \xi)+g(z)=0,
$$

где $\xi=c_{0} t-x, c_{0}-$ несущая скорость, вытекающая из начальной гипрерболической системы. Нелинейные функции $f(z)$ и $g(z)$ определяют 
роль ионного тока и диссипации и вызывают возможное усиление импульса до стационарного профиля. Вывод уравнения (1.3) и результаты численного эксперимента представлены в $\left[{ }^{10-12}\right]$. Решение получено в виде апериодической уединенной волны, что соответствует экспериментальным данным. Стационарный профиль такой аперигдической волны описывается теперь уравнением

$$
z^{\prime \prime}+f(z) z^{\prime}+\theta^{-1} g(z)=0,
$$

где $(\ldots)^{\prime}=d / d \eta, \eta=x+\theta \xi$, а параметр $\theta$ определяет действительную скорость $c$ стационарной волны $\left(c=\theta c_{0}(\theta-1)^{-1}\right)$. Как и в модели ФитцХью-Нагумо, опишем ионный ток кубическим полиномом. В результате уравнение (1.4) получит следующий вид:

$$
z^{\prime \prime}+\left(b_{0}+b_{1} z+b_{2} z^{2}\right) z^{\prime}+b_{3} z=0
$$

где $b_{0}, b_{1}, b_{2}, b_{3}$ - постоянные. Уравнение (1.5) принадлежит к классу уравнений Льенара $\left[{ }^{13}\right]$. Поведение его решения зависит от параметров и, в частности, от знаков корней $z_{1}$ и $z_{2}$ квадратного уравнения

$$
b_{2} z^{2}+b_{1} z+b_{0}=0 \text {. }
$$

Если $b_{3}>0$ и $z_{1}=-z_{2} \neq 0$, то (1.5) называется уравнением Ван дер Поля [ $\left.{ }^{1}\right]$; если $b_{3}>0, b_{2}<0, b_{1}>0, b_{0}>0$, т. е. $z_{1}<0, z_{2}>0$, то (1.5) описывает работу лампового генератора с мягким режимом $\left[{ }^{14}\right]$. В обоих случаях известно достаточно полное качественное поведение фазовых траекторий.

Если (1.5) моделирует стационарный профиль импульса в нервном волокне в виде апериодической уединенной волны, то по физическим соображениям на параметры уравнения (1.5) и корни квадратного уравнения (1.6) нужно налагать следующие ограничения:

$$
b_{0}>0, \quad b_{1}<0, \quad b_{2}>0, \quad b_{3}>0 ; \quad z_{1}>0, \quad z_{2}>0, \quad z_{1} \neq z_{2} .
$$

Исчерпывающий качественный анализ уравнения (1.5) при условиях (1.7), по данным авторов, отсутствует. В следующем разделе стандартными методами (см. напр., $\left.\left[{ }^{15}\right]\right)$ исследуется поведение траектории вблизи особой точки. В третьем разделе доказывается отсутствие предельного цикла. Отметим, что в $\left[{ }^{16}\right]$ это делается при дополнительном условии $0<b_{0}<2$ с учетом $b_{3}=1$.

2. Перепишем уравнение (1.5) в виде системы

$$
\begin{aligned}
& d z / \mathrm{dt}=y, \\
& d y / \mathrm{dt}=-b_{3} z-\left(b_{0}+b_{1} z+b_{2} z^{2}\right) y .
\end{aligned}
$$

Если $b_{3} \neq 0$, то начало координат $O(0,0)$ - единственная (простая) особая точка системы. При $b_{3}=0$ начало координат является сложным состоянием равновесия. В дальнейшем считаем, что $b_{3} \neq 0$.

Характер фазовой траектории вблизи начала координат определяется соответствующей линейной системой

$$
\begin{aligned}
& d z / \mathrm{dt}=y, \\
& d y / \mathrm{dt}=-b_{3} z-b_{0} y .
\end{aligned}
$$

Если $b_{3}<0$, то особая точка является седлом. По физическим соображениям $b_{3}>0$ (см. (1.7)), и тогда $O(0,0)$ - либо устойчивый (неустойчивый) узел, либо устойчивый (неустойчивый) фокус, в зависимости от знака дискриминанта $\Delta=b_{0}{ }^{2}-4 b_{3}$; если $\Delta>0$, то - узел, если $\Delta<0$, то - фокус (см. рис. 1). Отметим, что в $\left[{ }^{10,12}\right]$ такого различения фокуса и узла еще не отмечалось. Результаты, приведенные на рис. 1, разумеется, известны раньше [ $\left.{ }^{17}\right]$. 


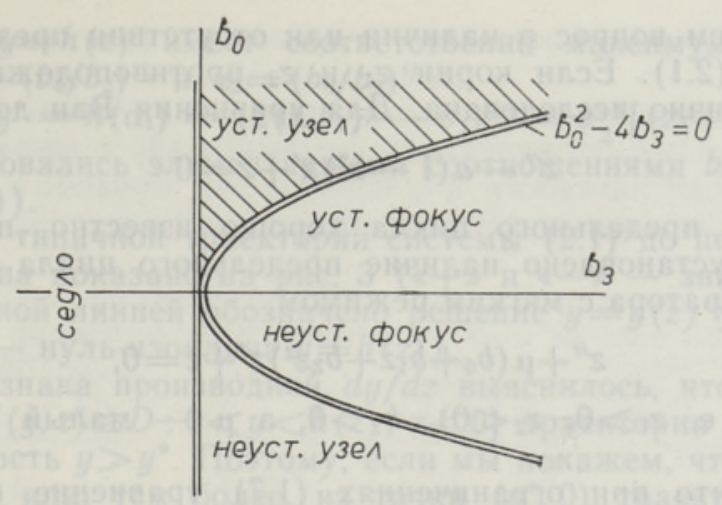

Рис. 1. Характер особой точки в зависимости от $b_{0}, b_{3}$. Двойная линия отмечает границы областей.

Физическому содержанию рассматриваемой задачи соответствует случай устойчивого узла, т. е. области $\Delta>0$ и $b_{0}>0$ (на рис. 1 заштрихована). Любая траектория стремится к началу координат по одной из двух направлений, которые определяются корнями характерного уравнения линейной системы (2.2). Другими словами, пусть $z(t)$ и $y(t)-$ решение уравнения (2.1). Тогда $\quad \lim _{t \rightarrow \infty} z(t)=0, \lim _{t \rightarrow \infty} y(t)=0$ и $\lim _{t \rightarrow \infty} y(t) / z(t)=k, \quad$ где $k-$ корень уравнения $k^{2}+b_{0} k+b_{3}=0$.

${ }_{t \rightarrow \infty}$ Зафиксируем $b_{3}>0$ и $b_{2}>0$. Плоскость параметров $b_{0}$ и $b_{1}$ с указанием характера особой точки показана на рис. $2\left(z_{1}\right.$ и $z_{2}$ - по-прежнему корни уравнения (1.6)). Для заданной задачи интерес представляет случай положительных корней $z_{1}$ и $z_{2}$, т. е. значения параметров из области с двойной штриховкой.

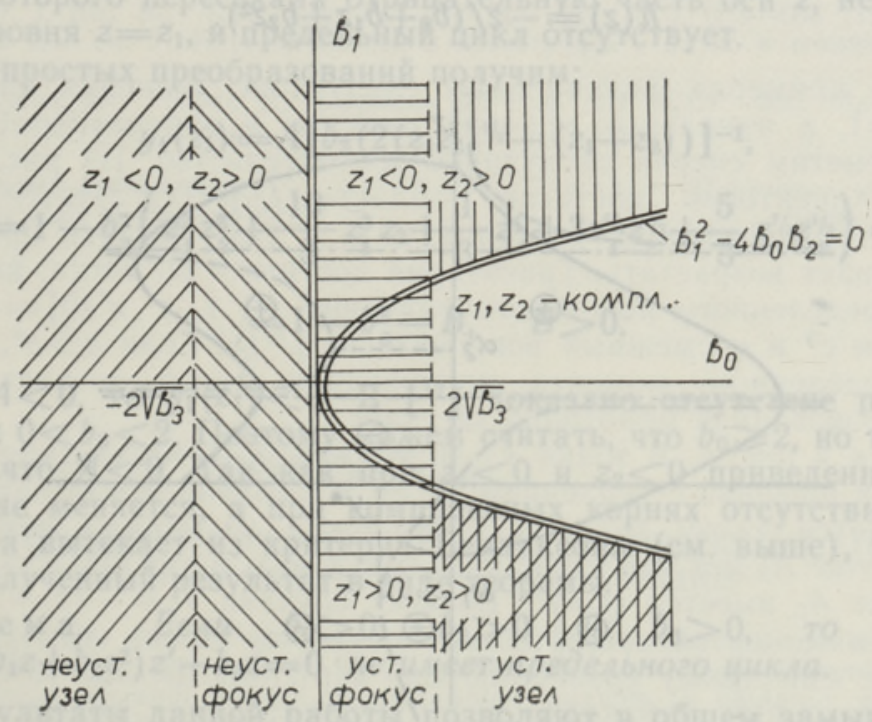

Рис. 2. Характер особой точки в зависимости от $b_{0}, b_{1}$. Двойная линия отмечает границы областей с различными корнями. 
3. Исследуем вопрос о наличии или отсутствии предельных циклов для системы (2.1). Если корни $z_{1}$ и $z_{2}$ противоположных знаков, то проблема частично исследована. Для уравнения Ван дер Поля

$$
z^{\prime \prime}-a\left(1-z^{2}\right) z^{\prime}+z=0
$$

существование предельного цикла хорошо известно при всех $a>0$ $\left[{ }^{1,14}\right]$. Также установлено наличие предельного цикла для уравнения лампового генератора с мягким режимом

$$
z^{\prime \prime}+\mu\left(b_{0}+b_{1} z+b_{2} z^{2}\right) z^{\prime}+z=0,
$$

где $b_{0}<0$ (т. е. $\left.z_{1}>0, z_{2}<0\right), b_{2}>0$, а $\mu$ - малый параметр ([ $\left.{ }^{14}\right]$ c. 703$)$.

Покажем, что при ограничениях (1.7) уравнение (2.1) не имеет предельного цикла. Так как изменением масштаба можно всегда один параметр нормировать, то для определенности зафиксируем $b_{3}=1$. Пусть также $z_{1}<z_{2}$.

Так как единственная особая точка $(2.1)$ - начало координат, то предельный цикл должен его охватывать. Рассмотрим уравнение второго порядка в общем виде: $z^{\prime}=P(z, y), y^{\prime}=Q(z, y)$. В области, где выражение $P_{z}+Q_{y}$ сохраняет знак, не может существовать предельных циклов (критерия Бендиксона $\left.\left[{ }^{15}\right]\right)$. В данном случае

$$
P_{z}+Q_{y}=-\left(b_{0}+b_{1} z+b_{2} z^{2}\right) \text {. }
$$

Следовательно, в области $z<z_{1}$ нет предельного цикла и всякий предельный цикл должен пересекать уровень $z=z_{1}$. Отметим, между прочим, что при комплексных корнях $z_{1}$ и $z_{2}$ отсюда сразу вытекает отсутствие предельного цикла на всей плоскости.

Исследуем подробнее поведение отдельной траектории на фазовой плоскости.

Из (2.1) получим следующее уравнение фазовой траектории $\left(b_{3}=1\right)$ :

$$
d y / d z=-\left(b_{0}+b_{1} z+b_{2} z^{2}\right)-z / y .
$$

Отсюда вытекает уравнение нуль-изоклины $y=h(z)$ :

$$
h(z)=-z /\left(b_{0}+b_{1} z+b_{2} z^{2}\right) .
$$

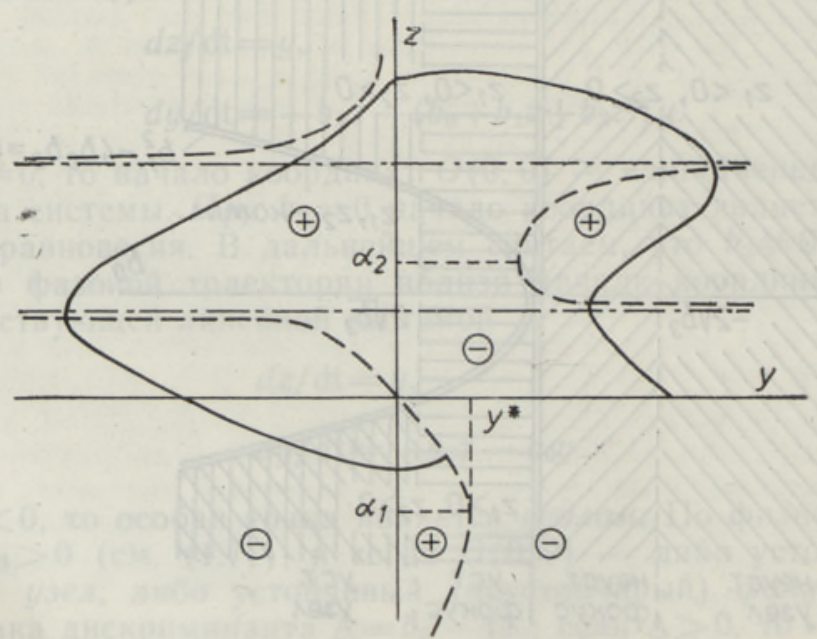

Рис. 3. Характерная фазовая плоскость. Сплошной линией обозначена траектория решения, прерывистыми - нуль-изоқлины, штрих-пунктирными - асимптоты к ним, 
Функция $y=h(z)$ имеет соответственно максимум и минимум в точках $\alpha_{1}=-\left(b_{0} / b_{2}\right)^{1 / 2}$ и $\alpha_{2}=\left(b_{0} / b_{2}\right)^{1 / 2}$.

Обозначим $y^{*}=h\left(\alpha_{1}\right)=\left(2\left(b_{0} b_{2}\right)^{1 / 2}-b_{1}\right)^{-1}=b_{2}^{-1}\left[\left(z_{1}+z_{2}\right)+2\left(z_{1} z_{2}\right)^{1 / 2}\right]^{-1}$ (мы воспользовались элементарными соотношениями $b_{0}=b_{2} z_{1} z_{2}$ и $b_{1}=$ $\left.=-b_{2}\left(z_{1}+z_{2}\right)\right)$.

Поведение типичной траектории системы (2.1) до пересечения нульизоклины слева показано на рис. 3 («+» и «-»- знаки производной $d y / d z$; сплошной линией обозначено решение $y=y(z)$ уравнения $(2.1)$, прерывистой - нуль-изоклина $y=h(z))$.

C учетом знака производной $d y / d z$ выяснилось, что выходящая из области $D=\{(y, z) \in D: 0<y<h(z), z<0\}$ траектория не может выйти на полуплоскость $y>y^{*}$. Поэтому, если мы покажем, что выходящая из точки $\left(y^{*}, \alpha_{1}\right)$ или, тем более, из точки $\left(y^{*}, 0\right)$ траектория уравнения (2.1) пересекает ось $z$ ниже точки $z=z_{1}$, то отсутствие предельного цикла установлено (так как в области $z<z_{1}$, в силу вышесказанного, предельного цикла нет).

Наряду с уравнением

$$
\left\{\begin{array}{l}
d y / d z=-\left(b_{0}+b_{1} z+b_{2} z^{2}\right)-z / y \\
y(0)=y^{*}
\end{array}\right.
$$

рассмотрим вспомогательное уравнение

$$
\left\{\begin{array}{l}
d y_{1} / d z=-\left(b_{0}+b_{1} z+b_{2} z^{2}\right)-z / y^{*} \\
y_{1}(0)=y^{*}
\end{array}\right.
$$

Очевидно, что в области $0<y<y^{*}, 0<z<z_{1}$ правые части уравнений (3.3) и (3.4) отрицательны и $|d y / d z|>\left|d y_{1} / d z\right|$. Поэтому в этой области выполняется $y(z)<y_{1}(z)$. Очевидно $\quad y_{1}(z)=y^{*}-b_{0} z-$ $-\left(b_{1} z^{2} / 2\right)-\left(b_{2} z^{3} / 3\right)-\left(z^{2} / 2 y^{*}\right)$.

Исследуем знак функции $y_{1}(z)$ в точке $z=z_{1}$. Если $y_{1}\left(z_{1}\right)<0$, то кривая $y_{1}(z)$ пересекает ось $z$ в промежутке $0<z<z_{1}$. Следовательно, тогда и решение $y=y(z)$ уравнения (3.3) не может пересекать ось $z$ выше точки $z=z_{1}$. Значит, в этом случае решение системы (2.1), траектория которого пересекала отрицательную часть оси $z$, не может достигать уровня $z=z_{1}$, и предельный цикл отсутствует.

После простых преобразований получим:

$$
\begin{gathered}
y_{1}\left(z_{1}\right)=A\left[b_{2}\left(2\left(z_{1} z_{2}\right)^{1 / 2}+\left(z_{1}+z_{2}\right)\right)\right]^{-1} \\
A=1-b_{2}^{2}\left(z_{1}^{2} z_{2}^{2}+\frac{10}{3} z_{1}^{3} z_{2}+\frac{1}{3} z_{1}^{4}+3 z_{1}^{5 / 2} z_{2}^{3 / 2}+\frac{5}{3} z_{1}^{7 / 2} z_{2}^{1 / 2}\right)= \\
=1-b_{0}^{2}-B, \quad B>0 .
\end{gathered}
$$

Если $A<0$, то $y_{1}\left(z_{1}\right)<0$. В $\left[{ }^{17}\right]$ доказано отсутствие предельного цикла при $0<b_{0}<2$. Поэтому можем считать, что $b_{0} \geqslant 2$, но тогда сразу получим, что $A<0$. Так как при $z_{1}<0$ и $z_{2}<0$ приведенное доказательство не меняется, а при комплексных корнях отсутствие предельного цикла вытекает из критерия Бендиксона (см. выше), то сформулируем полученный результат в виде теоремы.

Т еорем а. Если $b_{0}>0, \quad b_{2}>0 \quad u \quad b_{3}>0, \quad$ то $z^{\prime \prime}+\left(b_{0}+b_{1} z+b_{2} z^{2}\right) z^{\prime}+b_{3} z=0$ не имеет предельного циикла.

4. Результаты данной работы позволяют в общем замыкать исследование нвлинейного уравнения (1.4) типа Льенарда, в котором $f(z)$ имеет вид квадратичного полинома, a $g(z)$ - линейная функция. Подобно анализу элементарного линейного варианта $\left(f(z)=b_{0}\right)$ можно и 
здесь представить окончательный результат в виде наглядной карты решений $\left[{ }^{17}\right]$.

Так как нас интересует характер решения в зависимости от знаков корней $z_{1}$ и $z_{2}$, то представим карту решений на плоскости $z_{1} z_{2}$ (рис. 4). Характерные точки плоскости $z_{1} z_{2}$ обозначены через $A_{i}$ и соответствующие профили показаны рядом для каждой характерной точки.

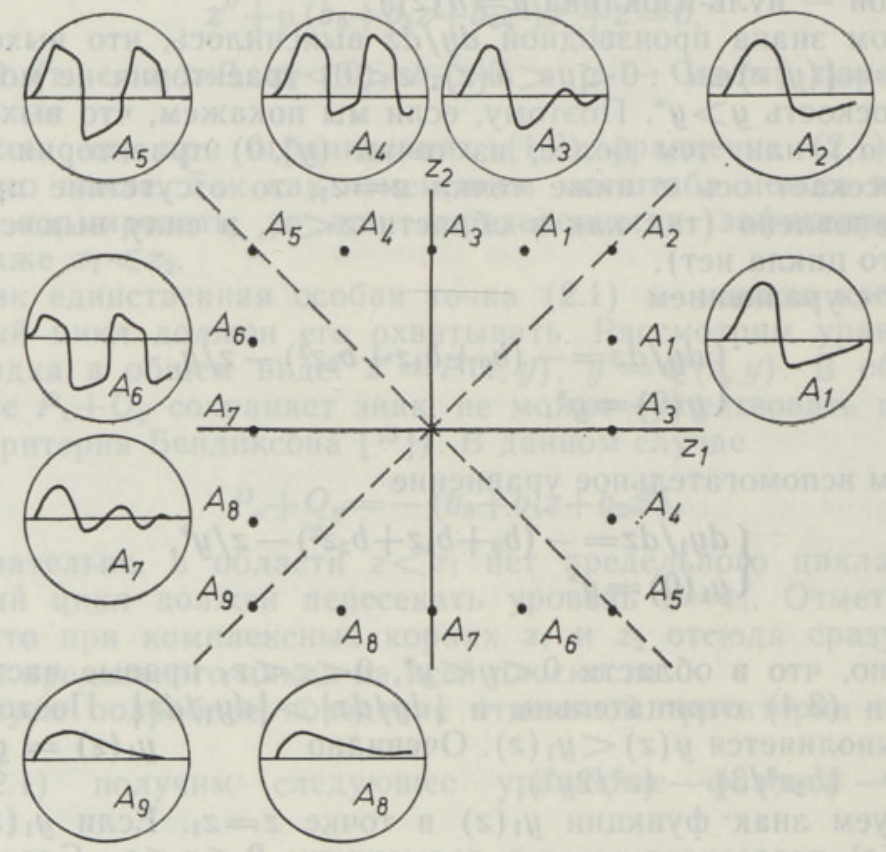

Рис. 4. Карта решений уравнения в зависимости от корней $z_{1}$ и $z_{2}$. Начальные условия $z(0)=0, z^{\prime}(0)=c>0$.

Во всех примерах фиксированы следующие значения параметров: $b_{2}=1, b_{3}=1$, а начальные условия приняты в виде $z\left(0 \mid=0, z^{\prime}(0 \mid=\right.$ $=c>0$. Отметим сперва следующее обстоятельство. Из рис. 3 видно, что если траектория достигает области $D_{1}=\left\{(y, z) \in D_{1}: y>h(z)\right.$, $\left.z_{1}<z<z_{2}\right\}$, то происходит усиление, т. е. в этой области производная $d y / d z$ начинает возрастать. Траектория достигает $D_{1}$ тогда, когда $z^{\prime}(0)$ больше определенного порогового значения $c^{*}$, т. е. $z^{\prime}\left(0 \mid=c>c^{*}\right.$ (об определении $c^{*}$ и о смежных вопросах см. $\left.\left[{ }^{18}\right]\right)$. Если $c>c^{*}$, то, начиная с определенного момента, производная резко возрастает, если $c<c^{*}$, то производная монотонно затухает.

Вернемся к рис. 4. В точке $A_{1}$ (в кружке $A_{1}$ ) указан типичный апериодический профиль с усилением $\left(c>c^{*}\right)$ в случае, когда начало координат является узлом $\left(b_{0} \geqslant 2\right)$. Если $b_{0}<2$, то начало координат фокус. В точке $A_{2}$ нет усиления (так как $z_{1}=z_{2}$ и нет области усиления $\left.D_{1}\right)$, в точке $A_{3}$ имеется фокус. Во всех случаях $b_{1}=-\left(z_{1}+z_{2}\right)<0$.

Вторая и четвертая четверть плоскости $z_{1} z_{2}$ (точки $A_{4}, A_{5}$ и $A_{6}$ ) соответствуют известному уравнению Ван дер Поля. Всегда $b_{0}<0$ и относительные величины положительных и отрицательных амплитуд зависят от знака $b_{1}$.

Различие в поведении профиля в точках $A_{3}$ и $A_{7}$ состоит в том, что в точке $A_{3}$ при $c>-b_{1}^{-1}$ возникает усиление (т. е. производная начй- 
нает возрастать), а при $c<-b_{1}^{-1}-$ монотонное затухание. В точке $A_{7}$ при $c>0$ затухание наблюдается всегда.

Профиль в точке $A_{8}$ является зеркальным отражением профиля точки $A_{1}$. Так как $z^{\prime}(0 \mid=c>0$, то образуется лишь вторая «половина» профиля $A_{1}$ (в области, где $z(\eta \mid<0)$. Если взять $z^{\prime}(0 \mid<0$, то получим весь профиль $A_{1}$ с обратным знаком. Аналогично связаны и профили $A_{9}$ и $A_{2}$.

Если $z_{1}$ и $z_{2}-$ комплексны, то процесс затухает без промежуточного усиления и в зависимости от величины $b_{0}$ в начале координат имеется либо узел, либо фокус.

\section{Л И Т Е Р А У Р А}

1. Скотт Э. Волны в активных и нелинейных средах в приложении к электронике. М., «Сов. Радио», 1977.

2. Марри Дж. Нелинейные дифференциальные уравнения в биологии. Лекции о моделях. М., «Мир», 1983.

3. Зыков B. C. Моделирование волновых процессов в возбудимых средах. М., «Наука», 1984.

4. Hodgkin, A. L. The Conduction of the Nervous Impulse. Liverpool University, 1964.

5. Scott, A. C. Neurophysics. New York, J. Wiley, 1977.

6. Nagumo, J., Arimoto, S., Yoshizawa, S. Proc. IRE, 50, $2061-2070$ (1962).

7. Колмогоров А. Н., Петровский И. Г., Пискунов Н. С. Вопр. кибернет., вып. 12, 3-30 (1975).

8. Lieberstein, H. M. Math. Biosci., 1, 45-69 (1967).

9. Engelbrecht, J. Proc. Roy. Soc. London, A 375, 195-209 (1981).

10. Энгельбрехт Ю. К. Биофизика, 30, 124-128 (1985).

11. Энгельбрехт Ю. К. В кн.: Вопросы нелинейной механики сплошной среды. Таллин, «Валгус», 1985, 119-131.

12. Engelbrecht, J. Wave Motion, 8, 93-100 (1986).

13. Reissig, R., Sansone, G., Conti, R. Qualitative Theorie nichtlinearer Differentialgleichungen. Roma, Cremonese, 1963.

14. Андронов A. A., Витт A. A,. Хайкин C. Э. Теория колебаний. М., «Физматгиз», 1959.

15. Баутин H. H., Леонтович $E$. A. Методы и приемы качественного исследования динамических систем на плоскости. М., «Наука», 1976.

16. Barbâlat, I. Bul. Sti. Acad. Rep. Pop. Romine, VII, № 3, 653-666 (1955)

17. Томпсон Дж. М. Т. Неустойчивости и катастрофы в науке и технике. М., «Мир», 1985.

18. Энгельбрехт Ю., Тобиас T., Кяэрманн М. Изв. АН ЭССР. Физ. Матем., 36, № 2, $177-185$ (1987).

Ннститут кибернетики

Академии наук Эстонской ССР
Поступила в редакцию $24 / \mathrm{X} 1986$

\section{J. ENGELBRECHT, $\overline{|T . T O B I A S|}$, Anu UUS}

\section{MITTEPERIOODILISE OKSIKLAINE LEVIMIST KIRJELDAVA LIÉNARD'I VORRANDI KVALITATIIVNE ANALOUS}

Närvikius leviva impulsi statsionaarse profiili kirjeldamiseks kasutatakse vastavast evolutsioonivõrrandist tuletatud $\left[{ }^{9,}{ }^{10}\right]$ Liénard'i võrrandi tüüpi diferentsiaalvõrrandit (1.5). On antud võrrandi lahendi kvantitatiivne analüüs ja näidatud, et kui esimest järku tuletise kordajaks oleva ruutpolünoomi lahendid $z_{1}$ ja $z_{2}$ on positiivsed, siis võrrandil piirtsüklit pole. Tasandil $z_{1} z_{2}$ on antud ka lahendite täielik kaart. 


\section{THE QUALITATIVE ANALYSIS OF THE LIENARD-TYPE MODEL EQUATION DESCRIBING THE APERIODIC PROFILE OF A SOLITARY WAVE}

Beside the common approach using the FitzHugh-Nagumo model, the special evolution equation (1.3) is used for describing the nerve pulse propagation $\left[{ }^{9,10}\right]$. The stationary profile is then determined by the Liénard-type second-order model equation (1.5). Its solution corresponds to the aperiodic profile of a solitary wave. The qualitative analysis of this equation and its solutions are presented. The phase portraits around the singular point are investigated and their distinctive features are determined in depen. dence on the coefficients of the equation (1.5). The main emphasis is devoted to the case $z_{1}>0, z_{2}>0, z_{1} \neq z_{2}$, where $z_{1}, z_{2}$ are the roots of the equation $b_{0}+b_{1} z+b_{2} z^{2}=0$. The following theorem is proved:

If $b_{0}>0, b_{2}>0$ and $b_{3}>0$, then the equation

$$
z^{\prime \prime}+\left(b_{0}+b_{1} z+b_{2} z^{2}\right) z^{\prime}+b_{3} z=0
$$

has no limit cycles.

For such an equation the full map of the solutions is presented in the plane $z_{1} z_{2}$. This analysis completes the study of the Liénard-type second-order nonlinear equations containing also the well-known case of the Van der Pol equation $\left(z_{1}=-z_{2} \neq 0\right)$. When the Van der Pol equation describes the periodical solution with a certain limit cycle, the general Liénard-type equation may lead to the aperiodic solutions needed for describing the solitary waves. 